\title{
Effect of wetting transition during multiphase displacement in porous media
}

\author{
Zhongzheng Wang, ${ }^{\dagger}, \ddagger$ Jean-Michel Pereira ${ }^{\ddagger}$ and Yixiang Gan*, ${ }^{*}$ \\ $\dagger$ School of Civil Engineering, The University of Sydney, NSW 2006, Australia \\ $\ddagger$ Navier, Ecole des Ponts, Univ Gustave Eiffel, CNRS, Marne-la-Vallée, France \\ E-mail: yixiang.gan@sydney.edu.au
}

\begin{abstract}
The effects of wettability on multiphase displacement in porous media have been studied extensively in the past, and the contact angle is identified as an important factor influencing the displacement patterns. At the same time, it has been found that effective contact angle can vary drastically in a time-dependent manner on rough surfaces due to Cassie-Wenzel wetting transition. In this study, we develop a theoretical model at the pore scale describing the apparent contact angle on rough interface as a function of time. The theory is then incorporated into the Lattice Boltzmann method for simulation of multiphase displacement in disordered porous media. A dimensionless time ratio, $D y$, describing the relative speed of wetting transition and pore invasion is defined. We show that the displacement patterns can be significantly influenced by $D y$, where more trapped defending ganglia are observed at large $D y$, leading to lower displacement efficiency. We investigate the mobilization of trapped ganglia through identifying different mobilization dynamics during displacement, including translation, coalescence, and fragmentation. Agreement is observed between the mobilization statistics and the total pressure gradient across a wide range of $D y$. Understanding the effect of wetting transition during multiphase displacement in porous media is of importance
\end{abstract}


for applications such as carbon geosequestration and oil recovery, especially for porous media where solid surface roughness cannot be neglected.

\section{INTRODUCTION}

Fluid-fluid displacement in porous media is an important phenomenon in many natural processes and engineering applications, including water infiltration into soils ${ }^{1}$, enhanced oil recovery ${ }^{2,3}$, and carbon geosequestration ${ }^{4,5}$. The displacement patterns are influenced by the characteristics of solid and liquid phases, including pore size distribution and topological disorder, fluid properties, and flow conditions ${ }^{6-13}$. When gravity is negligible, the multiphase displacement is governed by the interplay between capillary and viscous forces, which can be described by the capillary number $C a=\frac{\mu v}{\sigma}$, where $\mu$ is the viscosity of defending fluid, $v$ is the characteristic velocity of invading fluid, and $\sigma$ is the interfacial tension between the fluids. Besides, wettability, or contact angle, also has significant impacts on the displacement process. Here, the contact angle is measured within the invading fluid. When the contact angle of invading phase becomes smaller, the invasion front tends to be stabilized due to increased occurrence of cooperative pore filling events ${ }^{14-16}$, which leads to higher displacement efficiency. When the contact angle further decreases, however, the displacement efficiency may decrease due to corner flow ${ }^{12,17,18}$.

It has been found that the wettability of solids can vary in a wide range due to direct ionic bonding, Coulomb interactions, and precipitation ${ }^{19}$. A recent study by AlRatrout et $\mathrm{al}^{20}$ showed that the apparent contact angle is correlated with the surface roughness, being lower on rougher surfaces, which results from the accumulation of water in crevices. Mehmani et $\mathrm{al}^{21}$ investigated the effects of pore-scale roughness on displacement processes in sandstones, and they also found that increase in roughness results in smaller effective contact angle, which consequently stabilizes the displacement and facilitates the recovery process. However, in a study on two-phase flow displacement in rough fracture by Chen et $\mathrm{al}^{22}$, as 
the surface becomes rougher, an increase in apparent contact angle was observed during the multiphase displacement process. The relationship between apparent contact angle and surface roughness can be determined using Wenzel or Cassie-Baxter model, depending on the wetting states ${ }^{23-26}$. If the invading fluid is partially in contact with the surface, i.e., some defending phase is trapped in the crevices due to limited relaxation time in a relatively fast displacement process, the apparent contact angle will become larger according to CassieBaxter equation. If the invading fluid moves slowly such that it can completely fill the grooves on the surface, it is called Wenzel state, leading to smaller apparent contact angle for intrinsically water-wet media. The equilibrium contact angle can be determined by the modified Cassie-Baxter equation ${ }^{24-26}$ :

$$
\cos \theta_{\text {eq }}=r_{w} \phi \cos \theta_{0}+\phi-1,
$$

where $\phi \in[0,1]$ is the proportion of projected liquid-solid contact area (in the case of twofluid displacement in porous media, $\phi$ is the proportion of projected invading fluid-grain contact area), $r_{w}$ is the surface roughness ratio larger than or equal to 1 , defined as the total area divided by the apparent area, and $\theta_{0}$ is the intrinsic contact angle.

The aforementioned transition of wetting states, or Cassie-Wenzel wetting transition, has been investigated extensively through both experiments and simulations ${ }^{27-35}$. The energy barrier that needs to be overcome for the transition to take place is found to be dependent on geometry of micro-structures ${ }^{36-42}$, size of surface features ${ }^{43}$, hydrodynamic pressure ${ }^{44}$, line tension ${ }^{45}$, intrinsic contact angle ${ }^{31}$, and concentration of dissolved defending phase in the invading phase ${ }^{31}$. Larger energy barrier corresponds to increased stability of Cassie-Baxter state, leading to slower transition to Wenzel state. The typical time for wetting transition to complete ranges from seconds to days, depending on the aforementioned factors and liquid properties, including interfacial tension and viscosity. ${ }^{28-31}$ It has been also found that hierarchical surface structure can dramatically increase the stability of the Cassie state, 
slowing the transition ${ }^{46-48}$. On the other hand, external excitations, such as vibration can help overcome the energy barrier, accelerating the wetting transition ${ }^{49-51}$. Mishra et al ${ }^{27}$ demonstrated that the apparent contact angle changes from around $140^{\circ}$ to $60^{\circ}$ due to wetting transition on rough PDMS plates as time of contact increases. Seo et $\mathrm{al}^{31}$ experimentally showed a log-normal relation between intrinsic contact angle and the average time required for pore filling. As a result, the effective contact angle during multiphase fluids displacement in porous media with rough grains can be vastly influenced by the wetting state. The contact angle has an impact on the morphology of the invading fluid and the geometry of the menisci, which determines when, where, and whether a pore-filling event will take place. Usually the pore-filling events, e.g., two menisci merging, happen instantly. Since in contrast the time scale of the change in contact angle is not instantaneous, the local wettability is kept unchanged during pore-filling events. The available time for the progress of wetting transition is related to the local speed of pore invasion, which can be controlled by the injection velocity. Therefore, we hypothesize the existence of a competition between the progresses of pore invasion and wetting transition, which influences the effective contact contact angle during the advancement of invading fluid, consequently affecting the multiphase displacement patterns.

In this work, a theoretical model describing the local apparent contact angle for each individual grain as a function of time is proposed. This model is then incorporated into the Lattice Boltzmann method for simulation of multiphase displacement in porous medium, such that the wettability of each individual grain in the medium depends on the time of contact between the solid and invading fluid. To characterize the dynamic effect of wetting transition in multiphase displacement, we define a dimensionless parameter $D y=\frac{\tau_{W T}}{\tau_{P I}}$, where $\tau_{W T}$ and $\tau_{P I}$ are the characteristic times of wetting transition and pore invasion, respectively. Then, we study how the time-dependent behaviour of contact angle influences the multiphase displacement process in porous media. 


\section{METHODOLOGY}

\section{Media geometry and simulation method}

The medium is a rectangular domain filled with circular obstacles to simulate the solid phase (Fig.1(A)). Through varying the diameter of the obstacle $d$, the porosity of the medium can be controlled. Initially, these obstacles are regularly placed on a triangular lattice, and the spacial disorder is introduced by iterative Monte-Carlo movement of each obstacle with an apparent diameter $d_{\text {app }}=\lambda d$. In this study we fix $\lambda=1.2$. The detailed process has been explained in a previous study ${ }^{11}$. With the choice of a periodic domain with $x \in$ $[0.0433,3.8538]$ and $y \in[0,1]$ filled with 440 obstacles having a diameter of 0.06 , the overall porosity is $\Phi=0.674$.

The conventional Shan-Chen multiphase Lattice Boltzmann method with D2Q9 lattice is adopted for our 2D numerical simulations ${ }^{52,53}$. In this study, Bhatnagar-Gross-Krook (BGK) approach is preferred to Multi-Relaxation-Time (MRT) one due to extra computational resources and complexity required for the latter ${ }^{54}$. We choose a mesh size of $800 \times 3200$ $l u^{2}$ (lu: lattice unit) for the simulation area such that at least 10 lattices are in between the smallest throat to ensure the grid is fine enough ${ }^{55}$. The invading and defending fluids both have a density of $1 \mathrm{lu}$. The kinematic viscosity $\nu$ for both fluids is $0.1667 \mathrm{lu}$, leading to a viscosity ratio of 1 . The interfacial tension can be calculated using Young-Laplace equation, which is $\sigma=0.215 \mathrm{lu}$. The invading fluid is injected from the left with a constant velocity of $V_{\text {in }}=0.002 \mathrm{lu}$, leading to a capillary number $C a=\frac{V_{\text {in }} \mu_{\text {def }}}{\sigma}=0.0016$. Comparing with past literature ${ }^{10,16}$, this implies that the flow process is under viscous dominated regime. However, since both fluids have the same viscosity and the displacement is neither favourable (viscous stabilizing effect) nor unfavourable (viscous fingering), the influences of surface tension is still expected, which is shown in the next section. The pore scale Reynolds number $R e=\frac{\left(V_{\text {in }} / \Phi\right) \overline{L_{\mathrm{t}}}}{\nu}=0.57$, where $\overline{L_{\mathrm{t}}}=32 \mathrm{lu}$ is the average throat size. The outlet pressure at the right end is set to be a constant of $0.33 \mathrm{lu}$. Periodic boundary condition is applied at 
A $y$

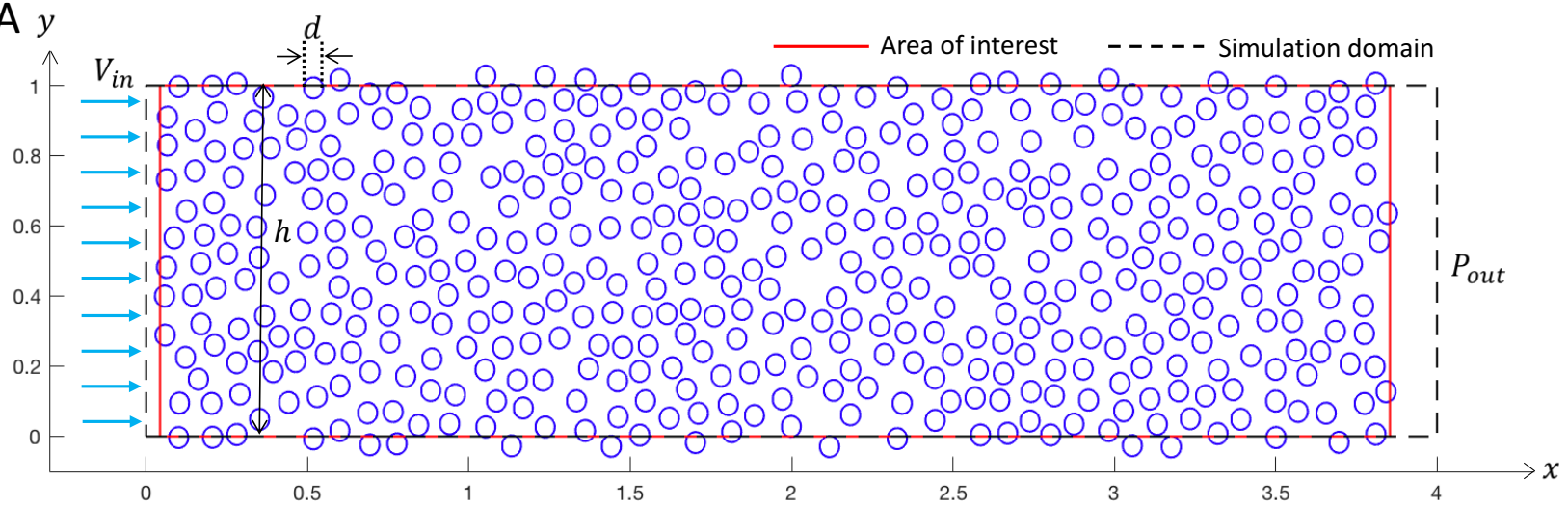

B

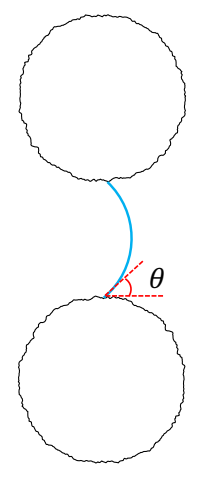

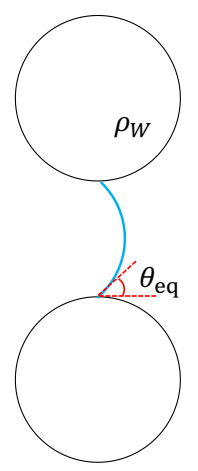

C
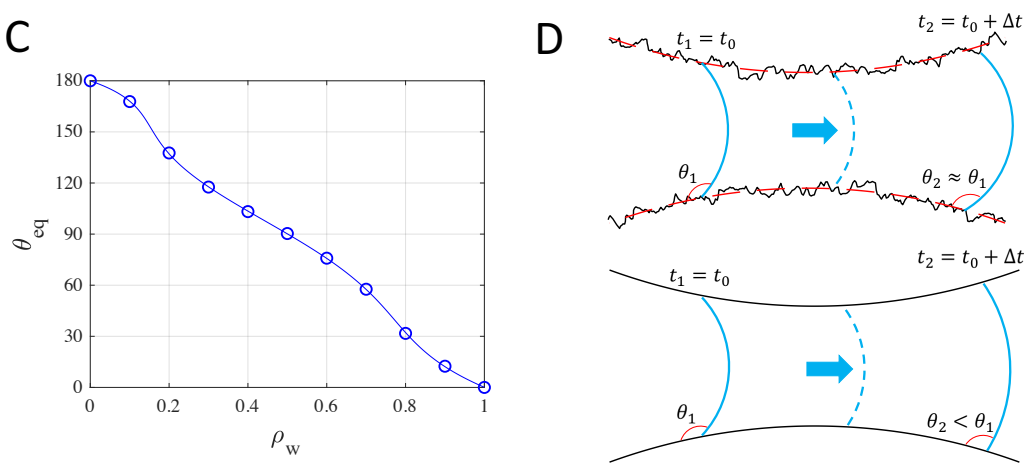

Figure 1: (A) Our simulated porous medium contains in total 440 circular obstacles with diameter $d=0.06$. The area enclosed by black-dashed line is the simulated area, while our results and analysis are based on the area of interest to exclude the empty space near the boundaries, which is marked by the red solid line. The invading fluid is injected from the left with a constant velocity $V_{\text {in }}$. Constant pressure boundary condition is set at the outlet. Periodic boundary condition is imposed at top and bottom of the simulation area. (B) Schematic of actual rough grains with an effective contact angle $\theta$ (left) and equivalent grains in simulations with an equilibrium contact angle $\theta_{\text {eq }}=\theta$ (right). (C) Equilibrium contact angle $\theta_{\mathrm{eq}}$ as a function of the fictitious wall density $\rho_{w}$. Blue circles represent simulation results, and solid line is the corresponding fitting curve using a 6th order polynomial. (D) Schematic showing the induced error in contact angle during invasion due to simplification on wettability of grains. Top: under constant velocity, with homogeneous surface features, the effective contact angle on rough surface is expected to be a constant. Bottom: the effective contact angle decreases due to the simplification, leading to underestimation of contact angle at latter stage of local pore invasion. 
top and bottom of the simulation area. The effective contact angle of spherical grains with rough surfaces is simulated by an equivalent contact angle on smooth grains as shown in Fig.1(B).

In the Shan-Chen multiphase scheme, the contact angle can be tuned by adjusting the fictitious wall density $\rho_{w}$, a parameter which influences the interaction strength between liquid and solid ${ }^{53,54,56}$. Fig.1(C) shows the equilibrium contact angle as a function of $\rho_{w}$. It can be seen that $\theta_{\text {eq }}$ monotonically decreases from $180^{\circ}$ to $0^{\circ}$ with the increase of $\rho_{w}$ from 0 to 1 . For simplicity, a 6th order polynomial is used for describing this relationship, which is shown as the solid line in Fig.1(C).

\section{Time-dependent wetting transition model}

In order to quantitatively relate the progress of wetting transition to time, Arrhenius equation with an activation energy being the adhesion energy is used in a recent study by Seo et $\mathrm{al}^{31}$. Here, we use a similar approach to consider the rate of Cassie-Wenzel wetting transition:

$$
\frac{\partial A_{\text {wenzel }}}{\partial t}=A_{\text {cassie }} \cdot k=\left(A_{\text {total }}-A_{\text {wenzel }}\right) \cdot k, \text { with } k=C e^{-\frac{E_{a}}{R T}}
$$

where $C$ (unit: $1 / \mathrm{sec}$ ) is the pre-exponential factor which can be a function of fluid viscosity and surface geometry, $E_{a}$ is the activation energy, which has been shown to be a function of intrinsic contact angle and interfacial tension ${ }^{31}, A_{\text {wenzel }}$, and $A_{\text {cassie }}$ are the surface areas under Wenzel state and Cassie state, respectively. After solving the partial differential equation we arrive at:

$$
\frac{A_{\text {wenzel }}}{A_{\text {total }}}=\phi=1+\left(\phi_{0}-1\right) e^{-k t}
$$

where $\phi_{0}$ is the initial liquid-solid contact fraction at $t=0$. It should be pointed out that 3 distinct regimes have been identified during wetting, including (1) rapid spreading stage after several milliseconds of initial contact $^{57,58}$, (2) grooves filling stage controlled by local forces, which is the focus in the current study, and (3) evaporation and condensation stage ${ }^{31}$. 
Eqn.(3) concerns the second stage, therefore it is appropriate to assume a non-zero $\phi_{0}$ after the first stage. The parameter $k$ can be regarded as a transition rate, which is inversely proportional to the characteristic time of wetting transition $\tau_{W T}=1 / k$, then we have

$$
\phi=1+\left(\phi_{0}-1\right) e^{-\frac{t}{\tau_{W T}}} .
$$
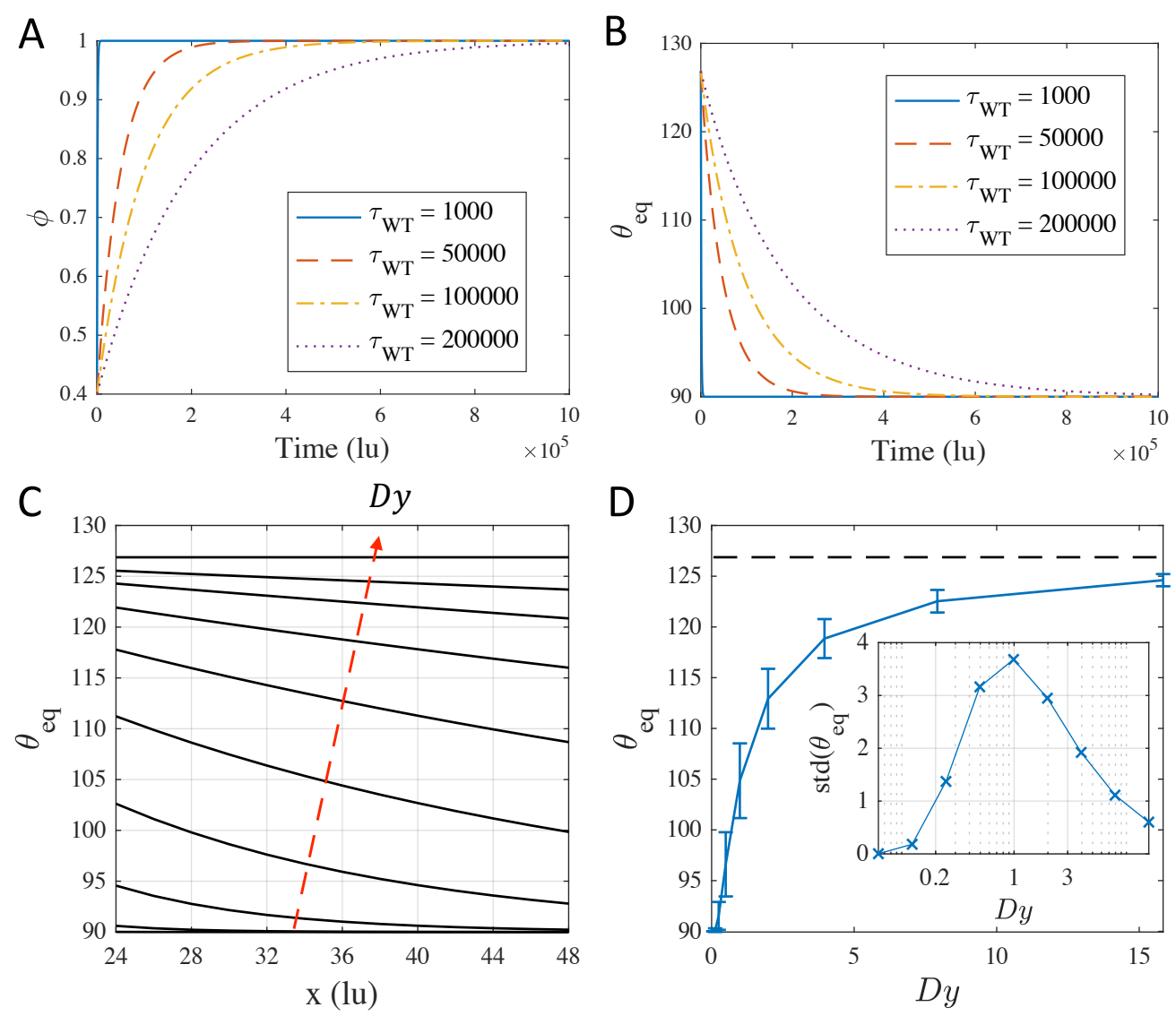

Figure 2: Time dependence of (A) Liquid-solid contact proportion $\phi$, (B) equilibrium contact angle $\theta_{\text {eq }}$, under different characteristic times of wetting transition $\tau_{W T}$. Note that time is in lattice unit (lu). (C) Equilibrium contact angle "experienced" by the invasion front along the latter half of particle $(d=48 \mathrm{lu})$. The red-dashed arrow indicates the direction of increasing $D y=\{0.1,0.2,0.4,0.8,1.6,3.2,6.4,12.8, \infty\}$. (D) The average equilibrium contact angle $\theta_{\text {eq }}$ as a function of $D y$, the errorbar indicates the standard deviation. The black-dashed line shows the value of $\theta_{\text {eq }}$ with $D y=\infty$ with a standard deviation of zero. The insert shows the standard deviation of $\theta_{\text {eq }}$ vs $D y$ in a semi-log plot.

Fig.2(A) shows the evolution of $\phi$ with an initial solid-liquid contact fraction $\phi_{0}=0.4$ 
for different characteristic times of wetting transition $\tau_{W T}$. Small values of $\tau_{W T}$ correspond to cases where fast groove filling events take place, and $\phi$ quickly approaches 1 , transiting to Wenzel state. As $\tau_{W T}$ increases, the speed of wetting transition slows down, but $\phi$ also ultimately approaches to 1 as time increases. For intrinsically hydrophobic rough surfaces with thermodynamically stable Cassie state, $\tau_{W T}=\infty$, which means the Cassie-Wenzel wetting transition will not occur spontaneously. Fig.2(B) shows the time evolution of equilibrium contact angle calculated from Eqn.(1) with a roughness ratio $r_{w}=1.1$. In all cases $\theta_{\text {eq }}$ decreases from $126.9^{\circ}$ to $90.2^{\circ}$. Note that, as mentioned previously, the value of the characteristic time of wetting transition $\tau_{W T}$ is a function of both surface features and fluid properties. However, in the current work, to limit our investigation to the effects of wetting transition during multiphase displacement, we do not aim to formulate theories on the characteristic time of wetting transition. Instead, $\tau_{W T}$ are prescribed, covering a wide range of values.

To incorporate the Eqn.(4) into the Lattice Boltzmann method, for each individual grain, the fictitious wall density is updated as a function of contact time $t_{\mathrm{c}}$ iteratively. The contact time can be calculated by $t_{\mathrm{c}}=T_{i}-T_{i}^{c}$, where $T_{i}$ and $T_{i}^{c}$ are the current time step and the time step when the invading fluid comes into contact with the grain, respectively. The event of contact is detected by scanning all peripheral lattices of a grain. A value of 0 is adopted for $t_{\mathrm{c}}$ when there is no contact. Fig.2(B) shows how the equilibrium contact angle changes with solid-liquid contact time $t_{c}$ at each individual grain for different characteristic time of wetting transition. This implies that the effective equilibrium contact angles can be different along the invasion front, depending on the invasion velocity. For a faster invasion front, corresponding to the situation where invasion takes place quickly and the time available for wetting transition is limited, the equilibrium contact angle is larger. Therefore, the heterogeneity in the time-scale of pore invasion can be simulated during multiphase displacement, where an invasion front with faster velocity experiences a larger effective contact angle. It is important to point out that, in order to significantly reduce the computation cost, we assume 
the wettability of a single grain to be homogeneous, i.e., all the peripheral lattices of one grain are updated together and have the same value of $\rho_{w}$. Realistically, it is not the case since the wetting state of one single grain can vary depending on the location, i.e., the contact angle would only change in the immediate vicinity of the contact line such that freshly covered sections of the grain would initially have high corresponding contact angles. Therefore, this assumption leads to underestimation of contact angle for regions that have not been touched by invading fluid, while their neighbor lattices on the same grain have. However, when there is a large difference between the characteristic times of wetting transition and pore invasion, this error reduces significantly, which will be further discussed next. Note that, although in this work we focus on Cassie-Baxter wetting transition, other theories describing different mechanisms affecting the contact angles such as the use of surface-active agents (e.g., surfactants, nanoparticles, etc) can also be incorporated into the Lattice Boltzmann method using a similar approach, which is however beyond the scope of current work.

The rate of local pore invasion is quantified by defining the characteristic time of pore invasion:

$$
\tau_{P I}=\frac{d}{V_{\mathrm{in}} / \Phi}
$$

where $d=48 \mathrm{lu}$ is the grain diameter. With $V_{\text {in }}=0.002 \mathrm{lu}$ and $\Phi=0.674, \tau_{P I}$ is calculated to be $16164 \mathrm{lu}$. Then, the ratio of time scales of wetting transition and pore invasion $D y$ is defined as:

$$
D y=\frac{\tau_{W T}}{\tau_{P I}} .
$$

A large value of $D y$ represents cases where local pore invasion proceeds much faster than wetting transition, where defending fluid is trapped in the crevices of grains, leading to larger contact angle due to Cassie wetting state. While smaller values of $D y$ indicate fast wetting transition towards Wenzel state.

Since homogeneous roughness feature is assumed and Dy is fixed during each individual simulation, the same effective contact angle is expected during the whole displacement 
process, despite some local variations depending on the invasion dynamics. However, due to the assumption on the simplified wettability of each grain as mentioned before, instead of having similar equilibrium contact angle $\theta_{\mathrm{eq}}$, the $\theta_{\mathrm{eq}}$ that is actually "experienced" by the invasion front will tend to decrease as the invasion front advances along each individual grain, as shown in Fig.1(D). This error in contact angle may influence the pore-scale mechanisms during displacement, including burst, touch, and overlap ${ }^{11,18,59}$. Consider the rear side of one throat, i.e., after the invasion front passing through the minimum throat with adjacent grains and one of the pore-scale mechanisms is about to take place, Fig.2(C) shows the equilibrium contact angle at the invasion front along the latter half of the grain $(d=48$ lu), assuming a constant velocity $v=V_{\text {in }} / \Phi$. The red-dashed arrow shows the direction of increasing $D y=\{0.1,0.2,0.4,0.8,1.6,3.2,6.4,12.8, \infty\}$. It is observed that all $\theta_{\text {eq }}$ have the decreasing tendency except for infinite Dy. Fig.2(D) and the insert show the average and standard deviation of equilibrium contact angle $\theta_{\mathrm{eq}}$ as a function of $D y$, respectively. Clearly, the error reaches maximum at $D y \approx 1$, indicating a comparable time scale between wetting transition and pore invasion, and decreases as $D y$ moves away from 1. Therefore, we choose $\tau_{W T}=10^{4} \times\{0.1,5,10,20, \infty\}$, which gives the corresponding $D y \approx\{0.1,3,6,12, \infty\}$. Note that in order have a wide range of $D y$, it is also possible to fix $\tau_{W T}$ while changing $\tau_{P I}$ through changing, for example, the injection velocity $V_{\text {in }}$. Nevertheless, we choose to fix $\tau_{P I}$ because (1) the computation cost greatly increases with low $V_{\text {in }},(2)$ the numerical stability of simulation decreases with large $V_{\text {in }},(3)$ change in $V_{\text {in }}$ results in different Ca, which introduces extra variables such as the effect from the dynamic contact angle hysteresis due to viscous dissipation ${ }^{60-63}$. 

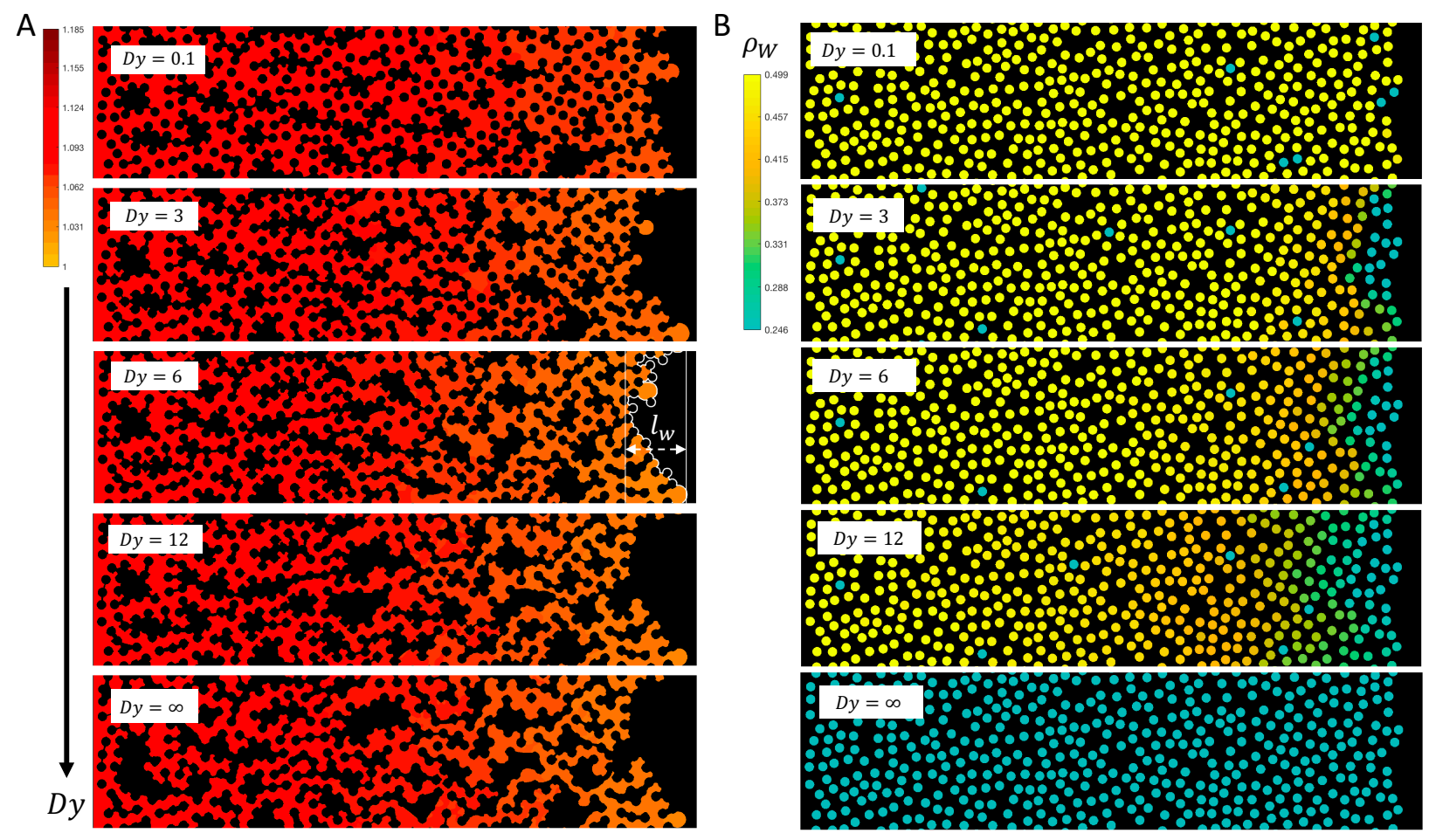

Figure 3: (A) Displacement patterns at percolation under varying Dy. The white solid curve at $D y=6$ marks front length $l_{1}$ along the invasion front. The length of dashed arrow is front width $l_{\mathrm{w}}$. The black color shows the location of defending fluid and grains, while red color shows the density fluctuation of invading fluid, which represents pressure variation in Lattice Boltzmann method. (B) Wettability distribution at percolation for different $D y$. Larger Dy indicates slower process of wetting transition compared with pore invasion. Colors represent values of the fictitious wall density $\rho_{w}$. 


\section{RESULTS AND DISCUSSION}

\section{Displacement efficiency}

The displacement patterns at percolation for 5 different $D y$ values are shown in Fig.3(A), which qualitatively demonstrate the influence of wetting transition speed on the invasion morphology. The trapped defending fluid and grains are represented by black color. Red color shows the invading fluid. With increase in $D y$, or larger characteristic time of wetting transition, it is observed that invasion front becomes less stable and larger amount of defending fluid is trapped inside the medium.
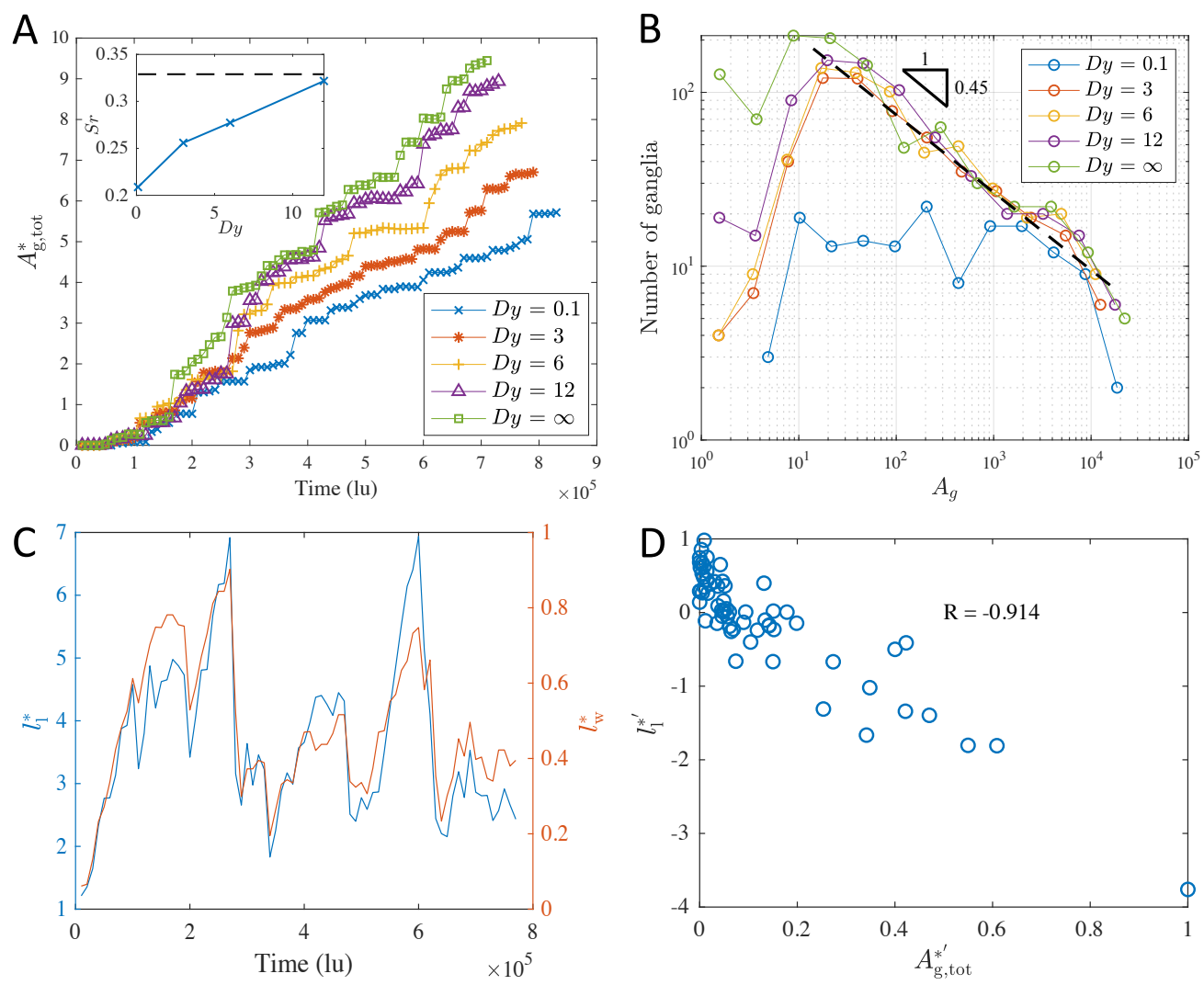

Figure 4: (A) Evolution of normalized total trapped ganglia area $A_{\mathrm{g} \text {, tot }}^{*}$ The insert shows the final residual saturation of defending fluids $S_{r}$ for different $D y$. The dashed line corresponds to the value of $S_{r}$ with $D y=\infty$. (B) Number of ganglia as a function of ganglia size $A_{\mathrm{g}}$. (C) Evolution of normalized interfacial length $l_{1}^{*}$ and width $l_{\mathrm{w}}^{*}$ for $D y=6$. (D) Correlation between the derivatives of $A_{\mathrm{g}, \text { tot }}^{*}$ and $l_{1}^{*}$ for $D y=6$. The correlation coefficient is $R=$ -0.914. (For other cases, $R=\{-0.858,-0.761,-0.909,-0.895\}$ for $D y=\{0.1,3,12, \infty\}$, respectively) 
In order to quantitatively reveal the effect of $D y$, Fig.4(A) shows the evolution of total area of trapped ganglia $A_{\mathrm{g}, \text { tot }}^{*}$, which is defined by $A_{\mathrm{g}, \mathrm{tot}}^{*}=A_{\mathrm{g}, \mathrm{tot}} /(d h)$, where $d$ and $h$ are the grain diameter and injection width in lattice units, respectively, and $A_{\mathrm{g} \text {,tot }}$ is the total area of trapped ganglia in lattice unit. Here, ganglia refer to the trapped clusters of defending fluid, although different names may be used according to the cluster size ${ }^{64}$. Stepwise increases in $A_{\mathrm{g}, \text { tot }}^{*}$ are observed, corresponding to individual trapping events. When the wetting transition progresses slowly during the displacement process (corresponding to large $D y$ ), the contact angle at pore scale becomes larger due to more defending fluids trapped in the surface grooves. This increase in effective contact angle suppresses cooperative pore filling events, promoting trapping ${ }^{16,65}$, consistent with past results. ${ }^{11-14,16}$ Moreover, the volume of trapped ganglia for cases with large $D y(D y=12$, slow wetting transition) can be very close to the one with $D y=\infty$ where wetting transition does not take place. However, from Fig.3(B), there are significant differences in the wettability distribution between $D y=12$ and $D y=\infty$ except the region near the invasion front. This implies that changes in contact angles behind invasion front do not have a significant impact on the total trapping volumes, although it may affect the redistribution of the trapped ganglia. Fig.4(A) also shows that the simulation time (or percolation time) for different $D y$ ranges from $7 \times 10^{5}$ to $8.5 \times 10^{5}$ time steps, which correspond to an actual time of around 20 hours using $16 \mathrm{CPUs}$. The insert shows the residual saturation of defending fluid $S_{r}$ for different $D y$. The black-dashed line represents the $S_{r}$ value with $D y=\infty$. It is worth noting that it is possible to observe further reduction in residual saturation for porous media due to the connectivity of the defending phase trapped in the crevices of the pore space. But this phenomenon can only take place after breakthrough. ${ }^{66}$

The interfacial length in lattice unit $l_{1}$ can be defined as the total front length between the defending fluid and the union of invading fluid and grains, as shown by the white-solid curve in Fig.3(A). The normalized value can be calculated as $l_{1}^{*}=l_{1} / h$. The interfacial width $l_{\mathrm{w}}$ is the span of $l_{1}$ in the direction of injection, indicated by the dashed double arrow. It can also be normalized by $l_{\mathrm{w}}^{*}=l_{\mathrm{w}} / h$. Fig.4(C) shows the change of interfacial length (blue curve) and 
width (red curve) during the displacement for the case with $D y=6$. It can be seen that they both fluctuate around a value while having the similar trend. Fig.4(D) shows the correlation between the derivatives of $A_{\mathrm{g}, \text { tot }}^{*}$ and $l_{1}^{*}$, which are denoted by $A_{\mathrm{g}, \text { tot }}^{*^{\prime}}$ and $l_{1}^{*^{\prime}}$, respectively. A correlation coefficient of $R=-0.914$ is found, indicating that for each trapping event, the total trapped area increases, while both $l_{1}$ and $l_{\mathrm{w}}$ decrease, smoothing the invasion front. The patterns observed in Fig.4(C,D) also appear similarly in other cases with different $D y$ $(R=\{-0.858,-0.761,-0.909,-0.895\}$ for $D y=\{0.1,3,12, \infty\}$, respectively).

\section{Ganglia size distribution}

Fig.4(B) shows the ganglia size distribution for different $D y$ at percolation. In the size range from 10 to $10^{4} \mathrm{lu}$, a power law relation is present between the number of ganglia and ganglia size for all simulation results except $D y=0.1$. In the case of $D y=0.1$, the effective contact angle is smaller than other cases such that fewer trapping events occur during displacement, consequently leading to fewer trapped ganglia. The value of the exponent of power-law relation, or the slope of the black-dashed line as in Fig.4(B), is associated with structure of the pore network, and a wide range of values have been observed ${ }^{67}$. In this case an exponent of -0.45 is seen, indicated by the black triangle. Note that there are upper and lower bounds for power-law relation of the distribution due to the limited size of simulation domain and the minimum pore size, respectively. One can notice the large deviations of ganglia number at small $A_{\mathrm{g}}$, where less trapping events occur at lower $D y$, implying that wetting transition facilitates the stable displacement.

\section{Ganglia mobilization}

We also look at the mobilization of trapped ganglia during the two-phase flow. The trapped defending phase can be mobilized due to the momentum transfer from the invading phase when viscous drag plays an important role ${ }^{64}$. Fig.5(A) shows the pressure distribution of invading fluid in lattice unit at percolation along the normalized length of area of interest $L$ 

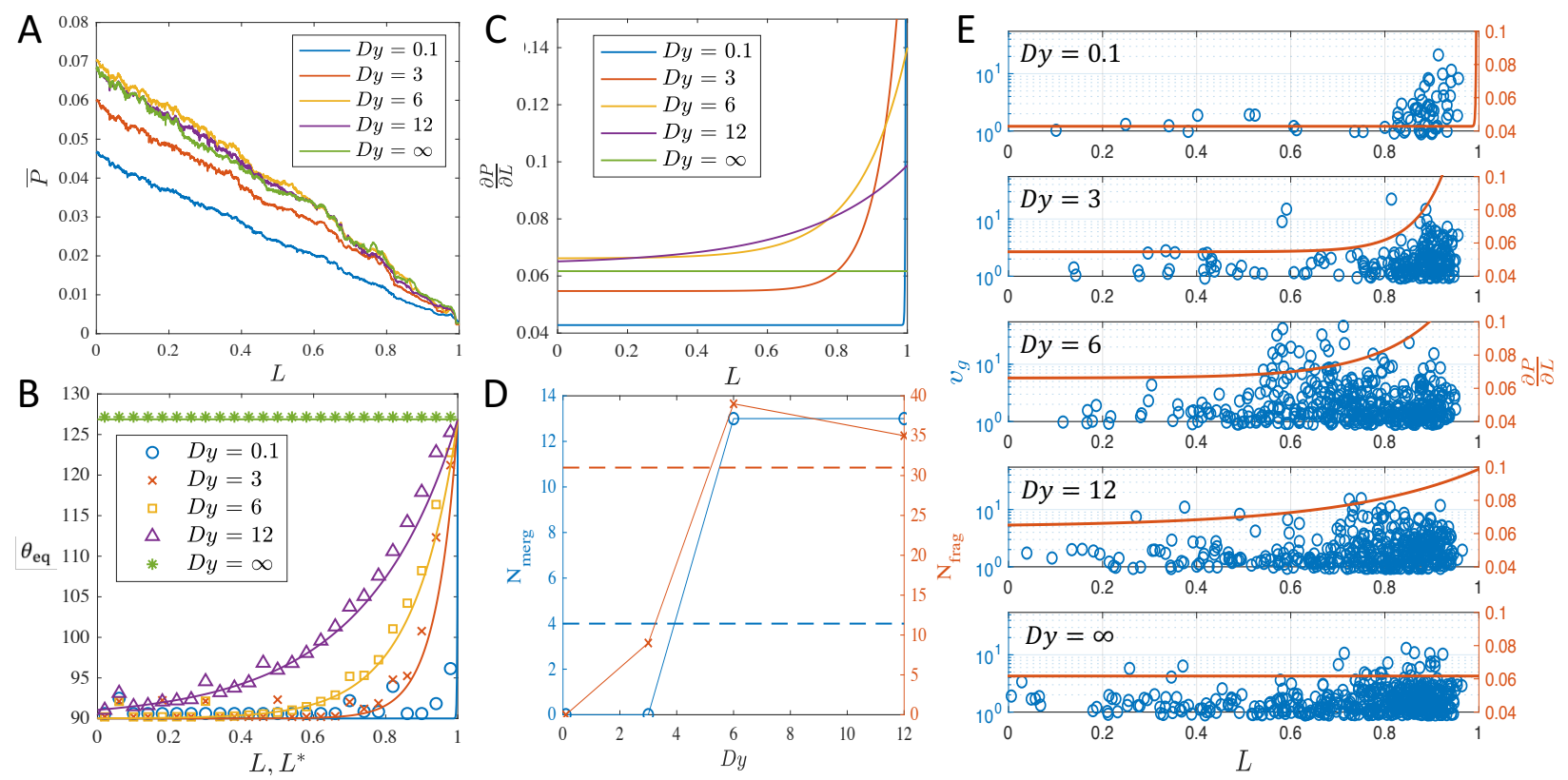

Figure 5: (A) Pressure distribution of invading fluid in lattice unit at percolation $\bar{P}$ in lattice unit along $x$-direction, which is averaged along $y$-direction. (B) Equilibrium contact angle $\theta_{\text {eq }}$ from theory (lines, plotted against $L$ ) and simulations (symbols, plotted against $L^{*}$ ) for different $D y$. (C) Total pressure gradient along $L$ for different $D y$. (D) Number of events of merging $\left(N_{\text {merg }}\right)$ and fragmentation $\left(N_{\text {frag }}\right)$ at different $D y$. The dashed lines correspond to values with $D y=\infty$. (E) Distribution of $v_{g}$, an indication of mobilized ganglia, along $L$ from simulations (blue circular circles), and the total pressure gradient in lattice unit from theory (red-solid lines). 
for different $D y$, where each value is averaged along the transverse direction. Overall, linear viscous pressure drop can be observed for all $D y$, with larger viscous pressure gradient for larger $D y$, although the difference is not significant for $D y>3$. However, viscous pressure alone is not sufficient for describing the mobilization of trapped ganglia, since the spacial wettability gradient can also exert pressures onto the defending phase due to the the timedependent local contact angles. Fig.3(B) shows the wettability distribution of the porous medium at percolation. The colors represent the values of the fictitious wall density $\rho_{w}$, which is a direct function of contact angles according to Fig.1(C). A wettability transition zone can be seen in the diagram and the width of the transition zone is affected by $D y$. For a more quantitative representation of the wettability distribution, symbols in Fig.5(B) shows the equilibrium contact angle $\theta_{\text {eq }}$ along $L$ with binsize of 0.04 , which is again averaged in the transverse direction from the simulation results. To compare with theory, a Lagrangian length scale needs to be introduced to convert time into spacial information:

$$
L^{*}=1-\frac{T_{i} \cdot V_{\mathrm{in}}}{\phi\left(1-S_{r}\right) X_{A O I}}
$$

where $X_{A O I}$ is the longitudinal length of area of interest in lattice unit. Note that $L^{*}=1$ represents the location of invasion front, whereas $L^{*}<1$ represents the location $1-L^{*}$ behind the invasion front. Then, the theoretical spacial wettability distribution is plotted with $L^{*}$ as solid lines in Fig.5(B). It can be seen that the theory matches well with the simulation results. Since the contact angle is larger near the front, from the perspective of defending fluid, this wettability gradient should facilitate the mobilization. To combine the pressure gradient from both viscous drag and interfacial tension, the total pressure gradient can be estimated as:

$$
\frac{\partial P}{\partial L}=\frac{\partial P_{\mathrm{vis}}}{\partial L}+\frac{2 \sigma}{\bar{L}_{\mathrm{t}}} \cdot \frac{\partial(\cos \theta)}{\partial L}
$$

where $\bar{L}_{\mathrm{t}}$ is the average throat size. Fig. $5(\mathrm{C})$ shows the total pressure gradient $\partial P / \partial L$ along $L$ for different $D y$. It is interesting to see that although the effective contact angle and 
total trapped defending ganglia are the largest for $D y=\infty$, due to the contribution from wettability change, the corresponding pressure gradient fails to dominate others, especially near the invasion front, whereas cases with $D y=6$ and $D y=12$ show larger pressure gradient, implying higher driving force for ganglia mobilization.

To verify our hypothesis, or the driving force for mobilizing ganglia, the distance of movement in lattice unit of each ganglion within a certain period (in this case 1000 time steps), or the characteristic velocity of ganglia $v_{\mathrm{g}}$ is plotted as blue circles in Fig.5(E) for different Dy. These movements of individual ganglion, or translation events, are direct reflection of ganglia mobilization. The corresponding total pressure gradient is also shown as red-solid line. We can see a concentrated mobilization events near the invasion front for all $D y$ at $L \approx 0.9$. This is simply a technical issue due to the fact that we have more data near the invasion front during the injection process. Qualitatively, it can be seen that the distribution of $v_{\mathrm{g}}$ reasonably agrees with the total pressure gradient $\partial P / \partial L$.

During the displacement process, apart from translation events, the trapped defending fluid can also experience other types of dynamics, including coalescence where two ganglia merge into one, and fragmentation where one ganglion becomes two. The number of occurrence of these events should provide an indication on the ganglia mobilization. Fig.5(D) shows the number of merging and fragmentation events for different $D y$. A non-monotonic relationship can be observed, and the trapped ganglia are more "active" with $D y=\{6,12\}$, consistent with the results from Fig.5(E). It is important to point out that the extent of influence due to change in contact angle can be different based on fluid property and flow conditions. For example, Holtzman and Segre ${ }^{16}$ showed that further decreases in contact angle below 60 degrees in capillary dominated regime does not have significant impact on the frequency of cooperative pore filling events. The impact of variation in contact angle is also found to be dependent on porosity and disorder ${ }^{68}$. Besides, during flow processes in viscous dominant regime with viscosity ratio much larger or smaller than 1 (either stable displacement or viscous fingering), the effect of interfacial tension will diminish compared 
with viscous pressure, consequently, the impact on displacement patterns due to change in contact angle is expected to decrease. Also note that, the events of ganglia motion, merging and fragmentation are reported up to the time of percolation. Longer simulations are likely to introduce additional events until steady state is reached. However, since the main purpose of the current work is to compare the ganglia mobilization for the same geometry to highlight the effect of $D y$, we did not further investigate the post-breakthrough behaviour.

\section{CONCLUSIONS}

The non-trivial correlation between the apparent contact angle and surface roughness in multiphase displacement is identified. We attribute the different behaviours of apparent contact angle to the relaxation time available for the progress of wetting transition, which is constrained by the speed of pore invasion. A theoretical model describing the time scale of Cassie-Wenzel wetting transition is developed, where the equilibrium contact angle can be directly related to time. Then the model is incorporated into the Lattice Boltzmann method for simulation of multiphase displacement in disordered porous media.

To quantitatively describe the competition between two characteristic pore-scale processes of wetting transition and pore invasion, a dimensionless time ratio, $D y$, describing the relative speed of these two mechanisms is defined. We show that the displacement patterns can be significantly influenced by $D y$ at percolation, where more trapped defending ganglia are observed at large $D y$ due to either fast invasion velocity or slow wetting transition, leading to lower displacement efficiency. Through studying different mobilization dynamics of trapped ganglia, including translation, coalescence, and fragmentation, agreement is shown between the mobilization statistics and the total pressure gradient, considering the effects of both viscous drag and capillary pressure.

Our study reveals the possible significant effect of injection velocity and surface roughness on the transient contact angle during displacement in porous media, which consequently 
affect displacement patterns. We also want to emphasize the potential underestimation of effective contact angle during displacement, as the experimentally measured correlation between contact angle and roughness after the displacement process has completed could be inaccurate due to subsequent wetting transition and fluid redistribution.

\section{Acknowledgement}

This work was financially supported by Australian Research Council (Projects DP170102886) and The University of Sydney SOAR Fellowship. YG acknowledges the financial support of Labex MMCD(ANR-11-LABX-022-01) for his stay at Laboratoire Navier at ENPC. This research was undertaken with the assistance of the HPC service at The University of Sydney.

\section{References}

(1) Lipiec, J.; Kuś, J.; Słlowińska-Jurkiewicz, A.; Nosalewicz, A. Soil porosity and water infiltration as influenced by tillage methods. Soil and Tillage Research 2006, 89, 210 220.

(2) Lake, L.; of Petroleum Engineers (U.S.), S. Fundamentals of Enhanced Oil Recovery; SPE continuing education; SPE, 1986.

(3) Blunt, M.; Fayers, F.; Orr, F. M. Carbon dioxide in enhanced oil recovery. Energy Conversion and Management 1993, 34, 1197 - 1204, Proceedings of the International Energy Agency Carbon Dioxide Disposal Symposium.

(4) Szulczewski, M. L.; MacMinn, C. W.; Herzog, H. J.; Juanes, R. Lifetime of carbon capture and storage as a climate-change mitigation technology. Proceedings of the National Academy of Sciences 2012, 109, 5185-5189.

(5) Matter, J. M.; Stute, M.; Snæbjörnsdottir, S. Ó.; Oelkers, E. H.; Gislason, S. R.; 
Aradottir, E. S.; Sigfusson, B.; Gunnarsson, I.; Sigurdardottir, H.; Gunnlaugsson, E.; Axelsson, G.; Alfredsson, H. A.; Wolff-Boenisch, D.; Mesfin, K.; Taya, D. F. d. l. R.; Hall, J.; Dideriksen, K.; Broecker, W. S. Rapid carbon mineralization for permanent disposal of anthropogenic carbon dioxide emissions. Science 2016, 352, 1312-1314.

(6) Lenormand, R.; Touboul, E.; Zarcone, C. Numerical models and experiments on immiscible displacements in porous media. Journal of Fluid Mechanics 1988, 189.

(7) Armstrong, R. T.; Georgiadis, A.; Ott, H.; Klemin, D.; Berg, S. Critical capillary number: Desaturation studied with fast X-ray computed microtomography. Geophysical Research Letters 2014, 41, 55-60.

(8) Yortsos, Y. C.; Xu, B.; Salin, D. Phase Diagram of Fully Developed Drainage in Porous Media. Phys. Rev. Lett. 1997, 79, 4581-4584.

(9) Rabbani, H. S.; Or, D.; Liu, Y.; Lai, C.-Y.; Lu, N. B.; Datta, S. S.; Stone, H. A.; Shokri, N. Suppressing viscous fingering in structured porous media. Proceedings of the National Academy of Sciences 2018, 115, 4833-4838.

(10) Holtzman, R. Effects of Pore-Scale Disorder on Fluid Displacement in PartiallyWettable Porous Media. Sci Rep 2016, 6, 36221.

(11) Wang, Z.; Chauhan, K.; Pereira, J.-M.; Gan, Y. Disorder characterization of porous media and its effect on fluid displacement. Phys. Rev. Fluids 2019, 4, 034305.

(12) Zhao, B.; MacMinn, C. W.; Juanes, R. Wettability control on multiphase flow in patterned microfluidics. Proceedings of the National Academy of Sciences 2016, 113, $10251-10256$.

(13) Trojer, M.; Szulczewski, M. L.; Juanes, R. Stabilizing Fluid-Fluid Displacements in Porous Media Through Wettability Alteration. Phys. Rev. Applied 2015, 3, 054008. 
(14) Cieplak, M.; Robbins, M. O. Influence of contact angle on quasistatic fluid invasion of porous media. Phys. Rev. B 1990, 41, 11508-11521.

(15) Jung, M.; Brinkmann, M.; Seemann, R.; Hiller, T.; Sanchez de La Lama, M.; Herminghaus, S. Wettability controls slow immiscible displacement through local interfacial instabilities. Phys. Rev. Fluids 2016, 1, 074202.

(16) Holtzman, R.; Segre, E. Wettability Stabilizes Fluid Invasion into Porous Media via Nonlocal, Cooperative Pore Filling. Phys. Rev. Lett. 2015, 115, 164501.

(17) Ran, H.; Jiamin, W.; Zhibing, Y.; Yi-Feng, C.; Tetsu, T. Wettability and Flow Rate Impacts on Immiscible Displacement: A Theoretical Model. Geophysical Research Letters 2018, 45, 3077-3086.

(18) Primkulov, B. K.; Talman, S.; Khaleghi, K.; Rangriz Shokri, A.; Chalaturnyk, R.; Zhao, B.; MacMinn, C. W.; Juanes, R. Quasistatic fluid-fluid displacement in porous media: Invasion-percolation through a wetting transition. Phys. Rev. Fluids 2018, 3, 104001.

(19) Morrow, N. R. Wettability and Its Effect on Oil Recovery. Journal of Petroleum Technology 1990, 42, 1476-1484.

(20) AlRatrout, A.; Blunt, M. J.; Bijeljic, B. Wettability in complex porous materials, the mixed-wet state, and its relationship to surface roughness. Proceedings of the National Academy of Sciences 2018, 115, 8901-8906.

(21) Mehmani, A.; Kelly, S.; Torres-Verdín, C.; Balhoff, M. Residual oil saturation following gas injection in sandstones: Microfluidic quantification of the impact of pore-scale surface roughness. Fuel 2019, 251, 147 - 161.

(22) Chen, Y.-F.; Wu, D.-S.; Fang, S.; Hu, R. Experimental study on two-phase flow in 
rough fracture: Phase diagram and localized flow channel. International Journal of Heat and Mass Transfer 2018, 122, 1298 - 1307.

(23) Wenzel, R. N. Resistance of solid surfaces to wetting by water. Industrial $\mathcal{E}$ Engineering Chemistry 1936, 28, 988-994.

(24) Cassie, A. B. D.; Baxter, S. Wettability of porous surfaces. Trans. Faraday Soc. 1944, $40,546-551$.

(25) Marmur, A. Wetting on Hydrophobic Rough Surfaces: To Be Heterogeneous or Not To Be? Langmuir 2003, 19, 8343-8348.

(26) Gao, N.; Yan, Y. Modeling Superhydrophobic Contact Angles and Wetting Transition. Journal of Bionic Engineering 2009, 6, 335-340.

(27) Mishra, H.; Schrader, A. M.; Lee, D. W.; Gallo, A.; Chen, S.-Y.; Kaufman, Y.; Das, S.; Israelachvili, J. N. Time-Dependent Wetting Behavior of PDMS Surfaces with Bioinspired, Hierarchical Structures. ACS Applied Materials $\&$ Interfaces 2016, 8, 81688174 .

(28) Moulinet, S.; Bartolo, D. Life and death of a fakir droplet: Impalement transitions on superhydrophobic surfaces. The European Physical Journal E 2007, 24, 251-260.

(29) Peters, A. M.; Pirat, C.; Sbragaglia, M.; Borkent, B. M.; Wessling, M.; Lohse, D.; Lammertink, R. G. H. Cassie-Baxter to Wenzel state wetting transition: Scaling of the front velocity. The European Physical Journal E 2009, 29, 391-397.

(30) Sbragaglia, M.; Peters, A. M.; Pirat, C.; Borkent, B. M.; Lammertink, R. G. H.; Wessling, M.; Lohse, D. Spontaneous Breakdown of Superhydrophobicity. Phys. Rev. Lett. 2007, 99, 156001.

(31) Seo, D.; Schrader, A. M.; Chen, S.-Y.; Kaufman, Y.; Cristiani, T. R.; Page, S. H.; 
Koenig, P. H.; Gizaw, Y.; Lee, D. W.; Israelachvili, J. N. Rates of cavity filling by liquids. Proceedings of the National Academy of Sciences 2018, 115, 8070-8075.

(32) Lopes, D. M.; Ramos, S. M. M.; de Oliveira, L. R.; Mombach, J. C. M. Cassie-Baxter to Wenzel state wetting transition: a 2D numerical simulation. RSC Adv. 2013, 3 , $24530-24534$.

(33) de Oliveira, L. R.; Lopes, D. M.; Ramos, S. M. M.; Mombach, J. C. M. Two-dimensional modeling of the superhydrophobic behavior of a liquid droplet sliding down a ramp of pillars. Soft Matter 2011, 7, 3763-3765.

(34) Zhao, X. Wetting transition of water on graphite: Monte Carlo simulations. Phys. Rev. B 2007, 76, 041402.

(35) Papadopoulos, P.; Mammen, L.; Deng, X.; Vollmer, D.; Butt, H.-J. How superhydrophobicity breaks down. Proceedings of the National Academy of Sciences 2013, $110,3254-3258$.

(36) Domingues, E. M.; Arunachalam, S.; Mishra, H. Doubly Reentrant Cavities Prevent Catastrophic Wetting Transitions on Intrinsically Wetting Surfaces. ACS Applied Materials $\mathfrak{E}$ Interfaces 2017, 9, 21532-21538.

(37) Amabili, M.; Giacomello, A.; Meloni, S.; Casciola, C. M. Intrusion and extrusion of a liquid on nanostructured surfaces. Journal of Physics: Condensed Matter 2016, 29, 014003.

(38) Arunachalam, S.; Das, R.; Nauruzbayeva, J.; Domingues, E. M.; Mishra, H. Assessing omniphobicity by immersion. Journal of Colloid and Interface Science 2019, 534, 156 $-162$.

(39) Bahadur, V.; Garimella, S. V. Preventing the Cassie-Wenzel Transition Using Surfaces with Noncommunicating Roughness Elements. Langmuir 2009, 25, 4815-4820. 
(40) Nosonovsky, M. Multiscale Roughness and Stability of Superhydrophobic Biomimetic Interfaces. Langmuir 2007, 23, 3157-3161.

(41) Nosonovsky, M.; Bhushan, B. Patterned Nonadhesive Surfaces: Superhydrophobicity and Wetting Regime Transitions. Langmuir 2008, 24, 1525-1533.

(42) Yoshimitsu, Z.; Nakajima, A.; Watanabe, T.; Hashimoto, K. Effects of Surface Structure on the Hydrophobicity and Sliding Behavior of Water Droplets. Langmuir 2002, 18, 5818-5822.

(43) Butt, H.-J.; Vollmer, D.; Papadopoulos, P. Super liquid-repellent layers: The smaller the better. Advances in Colloid and Interface Science 2015, 222, 104 - 109.

(44) Zheng, Q.-S.; Yu, Y.; Zhao, Z.-H. Effects of Hydraulic Pressure on the Stability and Transition of Wetting Modes of Superhydrophobic Surfaces. Langmuir 2005, 21, 1220712212.

(45) Bormashenko, E.; Whyman, G. On the Role of the Line Tension in the Stability of Cassie Wetting. Langmuir 2013, 29, 5515-5519.

(46) Bormashenko, E.; Gendelman, O.; Whyman, G. Superhydrophobicity of Lotus Leaves versus Birds Wings: Different Physical Mechanisms Leading to Similar Phenomena. Langmuir 2012, 28, 14992-14997.

(47) Wu, H.; Yang, Z.; Cao, B.; Zhang, Z.; Zhu, K.; Wu, B.; Jiang, S.; Chai, G. Wetting and Dewetting Transitions on Submerged Superhydrophobic Surfaces with Hierarchical Structures. Langmuir 2017, 33, 407-416.

(48) Michael, N.; Bhushan, B. Hierarchical roughness makes superhydrophobic states stable. Microelectronic Engineering 2007, 84, 382 - 386. 
(49) Bormashenko, E.; Pogreb, R.; Whyman, G.; Erlich, M. Cassie-Wenzel Wetting Transition in Vibrating Drops Deposited on Rough Surfaces: Is the Dynamic Cassie-Wenzel Wetting Transition a 2D or 1D Affair? Langmuir 2007, 23, 6501-6503.

(50) Bormashenko, E.; Pogreb, R.; Whyman, G.; Erlich, M. Resonance Cassie-Wenzel Wetting Transition for Horizontally Vibrated Drops Deposited on a Rough Surface. Langmuir 2007, 23, 12217-12221.

(51) Bormashenko, E.; Pogreb, R.; Stein, T.; Whyman, G.; Erlich, M.; Musin, A.; Machavariani, V.; Aurbach, D. Characterization of rough surfaces with vibrated drops. Phys. Chem. Chem. Phys. 2008, 10, 4056-4061.

(52) Shan, X.; Chen, H. Lattice Boltzmann model for simulating flows with multiple phases and components. Physical Review E 1993, 47, 1815-1819.

(53) Shan, X.; Doolen, G. Multicomponent Lattice-Boltzmann Model with Interparticle Interaction. Journal of Statistical Physics 1995, 81, 379-393.

(54) Kruger, T.; Kusumaatmaja, H.; Kuzmin, A.; Shardt, O.; Silva, G.; Viggen, E. M. LBM - the principles and methods; Springer, 2017.

(55) Raiskinmäki, P.; Shakib-Manesh, A.; Jäsberg, A.; Koponen, A.; Merikoski, J.; Timonen, J. Lattice-Boltzmann Simulation of Capillary Rise Dynamics. Journal of Statistical Physics 2002, 107, 143-158.

(56) Huang, H.; Thorne, D. T.; Schaap, M. G.; Sukop, M. C. Proposed approximation for contact angles in Shan-and-Chen-type multicomponent multiphase lattice Boltzmann models. Phys. Rev. E 2007, 76, 066701.

(57) Bird, J. C.; Mandre, S.; Stone, H. A. Short-Time Dynamics of Partial Wetting. Phys. Rev. Lett. 2008, 100, 234501. 
(58) Courbin, L.; Bird, J. C.; Reyssat, M.; Stone, H. A. Dynamics of wetting: from inertial spreading to viscous imbibition. Journal of Physics: Condensed Matter 2009, 21, 464127.

(59) Cieplak, M.; Robbins, M. O. Dynamical Transition in Quasistatic Fluid Invasion in Porous Media. Phys. Rev. Lett. 1988, 60, 2042-2045.

(60) Cox, R. G. The dynamics of the spreading of liquids on a solid surface. Part 1. Viscous flow. Journal of Fluid Mechanics 1986, 168, 169 - 194.

(61) Voinov, O. V. Hydrodynamics of wetting. Fluid Dynamics 1976, 11, $714-721$.

(62) Blake, T.; Haynes, J. Kinetics of liquidliquid displacement. Journal of Colloid and Interface Science 1969, 30, $421-423$.

(63) Petrov, P.; Petrov, I. A combined molecular-hydrodynamic approach to wetting kinetics. Langmuir 1992, 8, 1762-1767.

(64) Zarikos, I.; Terzis, A.; Hassanizadeh, S.; Weigand, B. Velocity distributions in trapped and mobilized non-wetting phase ganglia in porous media. Scientific Reports 2018, 8, 13228.

(65) Blunt, M. J. Multiphase Flow in Permeable Media: A Pore-Scale Perspective; Cambridge University Press, 2017.

(66) Blunt, M. J. Physically-based network modeling of multiphase flow in intermediate-wet porous media. Journal of Petroleum Science and Engineering 1998, 20, 117 - 125.

(67) Avendaño, J.; Lima, N.; Quevedo, A.; Carvalho, M. Effect of Surface Wettability on Immiscible Displacement in a Microfluidic Porous Media. Energies 2019, 12, 664.

(68) Hu, R.; Lan, T.; Wei, G.-J.; Chen, Y.-F. Phase diagram of quasi-static immiscible displacement in disordered porous media. Journal of Fluid Mechanics 2019, 875, 448-475. 
